\title{
QUASICONFORMAL MAPPINGS
}

\author{
Seppo Rickman
}

\section{Introduction}

This paper is an attempt to survey some features of the theory of quasiconformal mappings as it stands today. The modern development of the theory started in the fifties and since then the interest in the field has grown enormously. Much of this is due to the many applications and connections of quasiconformal mappings to other fields of mathematics. It is clear that in this short presentation I am able to consider only a part of the story and therefore I will limit myself to a few topics. The works by Ahlfors on the subject have had a great impact on the development of the theory and I have chosen the topics partly to emphasize this. However, I have left out one area where Ahlfors' influence has been important, namely the role of Teichmüller spaces. The reason for this is that this topic will be discussed in other papers in this volume.

There are many equivalent definitions for quasiconformal mappings. I will choose here the one that is conceptually the easiest, the so called metric definition. Let $D$ and $D^{\prime}$ be domains in the Euclidean $n$-space $R^{n}$ with $n \geq 2$ and let $f: D \rightarrow D^{\prime}$ be a homeomorphism. For $x \in D$ and $r>0$ such that the closed ball $\bar{B}(x, r)$ is in $D$ we write

$$
\begin{aligned}
& L(x, r)=\max _{|x-y|=r}|f(y)-f(x)|, \quad \ell(x, r)=\min _{|x-y|=r}|f(y)-f(x)|, \\
& H(x, r)=\frac{L(x, r)}{\ell(x, r)} \\
& H(x)=\limsup _{r \rightarrow 0} H(x, r) .
\end{aligned}
$$

We say that $f$ is quasiconformal if the linear dilatation $H(x)$ is uniformly bounded in $D$. In connection with this definition it is convenient (although it is not common terminology) to define $f$ to be $K$-quasiconformal with $1 \leq K<\infty$ if $f$ is quasiconformal and

$$
H(x) \leq K \quad \text { a. e. }
$$

The given definition for the Euclidean space generalizes to metric spaces, in particular to Riemannian $n$-manifolds. An important special case here is the 
one point compactification $\bar{R}^{n}=R^{n} \cup\{\infty\}$ of $R^{n}$ provided with the spherical metric. The concept of a quasiconformal mapping is clearly well defined also on $n$-manifolds with conformal structure without any a priori given metric, e. g. on Riemann surfaces. If not otherwise stated, we mean in the following the Euclidean case.

It is easy to give examples of large classes of quasiconformal mappings. If $f$ : $D \rightarrow D^{\prime}$ is a diffeomorphism, its restriction to any relatively compact subdomain is clearly quasiconformal. If $f$ is $L$-bilipschitz, it is $L^{2}$-quasiconformal. The converse is not true which is seen from the example $x \mapsto|x|^{\alpha-1} x$ where $\alpha>1$.

For $n=2$ a homeomorphism $f: D \rightarrow D^{\prime}$ is 1-quasiconformal if and only if $f$ is conformal or anticonformal. If $n \geq 3$, a 1 -quasiconformal mapping is always a restriction of a Möbius transformation. The last result is Liouville's theorem in a generalized form due to Gehring [G2] and Reshetnyak [R2].

It was Grötzsch who in the late 1920's was the first to consider quasiconformal mappings for $n=2$ and his main contribution [G12] consists of a study of extremal mappings between simple plane domains. The first appearance of the term quasiconformal is in 1935 in the famous paper on covering surfaces by Ahlfors [A1].

A crucial step forward was the discovery made by Teichmüller in the late 1930's [T1] that extremal quasiconformal mappings on closed Riemann surfaces lead to a connection with holomorphic quadratic differentials on the surfaces. The ideas of the theory of Teichmüller spaces were also given in [T1].

In the early articles certain smoothness properties were required for quasiconformal mappings. One of the main drawbacks in such definitions is the lack of compactness of families of $K$-quasiconformal mappings. In 1954 Ahlfors started in [A2] a systematic study of the class that we now call quasiconformal mappings and he cleared up part of Teichmüller's work. Already in 1938 Morrey [M2] had proved the important measurable Riemann mapping theorem (see Section 4), but people working in quasiconformal mappings had not paid attention to it. In the course of years Ahlfors, Bers, and their students have given a great contribution to the studies of Teichmüller spaces and other connections of quasiconformal mappings to complex analysis.

For dimensions $n \geq 3$ quasiconformal mappings were first treated in 1938 by Lavrent'ev [L1], but the systematic study began much later by Gehring and Väisälä in the early 1960's [G1], [G2], [V1]. Their study was preceded by an important article by Löwner [L4] on conformal capacity.

Recent development of quasiconformal theory has grown from the contribution of a large number of people in addition to the names listed above. To pick up two names, I want to mention Tukia and Sullivan. Sullivan, for example, has found many interesting applications and connections to other fields of mathematics. For some details, I refer to Section 5. I shall also include (Section 6) a discussion of the noninjective case, the theory of quasiregular mappings. These mappings have turned out to form an interesting generalization of the complex analytic functions 
to real $n$-dimensional space.

Good references for the 2-dimensional theory of quasiconformal mappings are the books by Ahlfors [A5] and Lehto and Virtanen [L2]. For general $n$ I recommend the book [V2] by Väisälä. Also the survey by Ahlfors [A6] and that by Gehring [G7] give an excellent picture of the subject.

\section{Local and global properties}

I shall start by describing how one passes from the uniform boundedness of the local notion, the linear dilatation $H(x)$, to global geometric properties. This involves integration in some sense which in turn requires certain regularity of the mapping.

2.1. Differentiability properties. Let now $f: D \rightarrow D^{\prime}$ be a $K$ quasiconformal mapping. One first shows that $f$ is $A C L$, that is, absolutely continuous on almost all lines parallel to the coordinate axes. The essential point in the proof is Lebesgue's theorem that an additive set function has a finite derivative a.e. It follows that $f$ has partial derivatives a.e., in fact, $f$ is even differentiable a.e. To prove this, first observe that for $x \in D$ we have $L(x, r)^{n} / r^{n} \leq c l(x, r)^{n} / r^{n} \leq c^{\prime} m\left(f B^{n}(x, r)\right) / r^{n}$ for some constants $c$ and $c^{\prime}$ when $r$ is small. Here $m$ is the Lebesgue measure. Lebesgue's theorem gives therefore

$$
L(x)=\limsup _{r \rightarrow 0} \frac{L(x, r)}{r}<\infty \quad \text { a.e. }
$$

Differentiability a.e. results then from the theorem of Rademacher-Stepanov.

A homeomorphism which is differentiable a.e. has a locally integrable Jacobian determinant $J_{f}$. If $f$ is differentiable at $x$ and $H(x) \leq K$, we have $\left|f^{\prime}(x)\right|^{n} \leq$ $K^{n-1}\left|J_{f}(x)\right|$. Here $\left|f^{\prime}(x)\right|$ is the operator norm of the derivative map $f^{\prime}(x)$. It follows that $\left|f^{\prime}(x)\right|$ is locally in $L^{n}$ which together with $A C L$ means that $f$ belongs to the class denoted by $A C L^{n}$. We obtain the following statement.

(A) If $f: D \rightarrow D^{\prime}$ is $K$-quasiconformal, then

(1) $f$ is differentiable a.e. ,

(2) $f$ is $A C L^{n}$,

(3) $\left|f^{\prime}(x)\right|^{n} \leq K^{n-1}\left|J_{f}(x)\right|$ a.e.

2.2. Modulus of a path family. Let $\Gamma$ be a family of nonconstant paths in $R^{n}$ and let $F(\Gamma)$ be the set of Borel functions $\rho: R^{n} \rightarrow[0, \infty]$ for which the line integral satisfies

$$
\int_{\gamma} \rho d s \geq 1
$$


for all locally rectifiable $\gamma \in \Gamma$. The central global notion in quasiconformal theory is the modulus

$$
M(\Gamma)=\inf _{\rho \in F(\Gamma)} \int_{R^{n}} \rho^{n} d m
$$

of $\Gamma$. The number $M(\Gamma)^{1 /(1-n)}$ is the extremal length of $\Gamma$.

The conditions (1)-(3) in (A) give enough regularity to prove the following result.

(B) If $f: D \rightarrow D^{\prime}$ is a homeomorphism that satisfies (1)-(3) in (A), then

$$
M(\Gamma) \leq K^{n-1} M(f \Gamma)
$$

whenever $\Gamma$ is a family of paths in $D$.

We can use (i) alone to show that both $f$ and $f^{-1}$ are quasiconformal and hence close the circle of reasoning:

(C) If $f: D \rightarrow D^{\prime}$ is a homeomorphism that satisfies (i) in (B), then $f$ and $f^{-1}$ are $C(n, K)$-quasiconformal where $C(n, K)$ depends only on $n$ and $K$.

I shall give a proof of the statement for $f$. Let $x \in D$ and let $r>0$ be small. Write $\ell=\ell(x, r), L=L(x, r)$, and let $\Gamma$ be the family of paths in $D$ which join the preimage of the sphere $S^{n-1}(f(x), \ell)$ to the preimage of the sphere $S^{n-1}(f(x), L)$. Since $f^{-1} S^{n-1}(f(x), \ell)$ and $f^{-1} S^{n-1}(f(x), L)$ both touch the sphere $S^{n-1}(x, r)$, we have an estimate of the form $M(\Gamma) \geq a_{n}>0$ where $a_{n}$ depends only on $n$. On the other hand, $M(f \Gamma) \leq \omega_{n-1}(\log (L / \ell))^{1-n}$ where $\omega_{n-1}$ is the $(n-1)$-measure of the unit sphere. These give with (i) an inequality of the form $H(x, r) \leq C(n, K)$, and (C) for $f$ is proved.

If $f: D \rightarrow D^{\prime}$ is $K$-quasiconformal, $f$ preserves zero sets, $J_{f}(x) \neq 0$ a.e., and

$$
m(f E)=\int_{E}\left|J_{f}\right| d m
$$

for measurable $E \subset D$. It follows then from (A)-(C) that $f^{-1}$ is also $K$ quasiconformal. If $g: D^{\prime} \rightarrow D^{\prime \prime}$ is $K^{\prime}$-quasiconformal, then $g \circ f$ is $K^{\prime} K$ quasiconformal.

2.3. Equivalent definitions. Let $f: D \rightarrow D^{\prime}$ be a homeomorphism. According to (A)-(C) $f$ is quasiconformal if and only if $f$ satisfies (1)-(3) in 2.1 for some $K$. This is often called the analytic definition. The conditions (1)-(3) are not independent for homeomorphisms. In fact, conditions (2) and (3) alone imply quasiconformality (if $f^{\prime}(x)$ is defined by means of the partial derivatives).

If $f: D \rightarrow D^{\prime}$ is $K$-quasiconformal, it not only satisfies (i) in (C), but also the double inequality

$$
K^{1-n} M(\Gamma) \leq M(f \Gamma) \leq K^{n-1} M(\Gamma) .
$$


Conversely, (ii) implies quasiconformality of $f$. The condition (ii) for a homeomorphism $f$ is called the geometric definition of quasiconformality. It also shows that the modulus of a path family is a conformal invariant. The geometric definition was given by Ahlfors for $n=2$ in [A2].

The double inequality (ii) is the most effective tool for quasiconformal mappings that applies in all dimensions. For example, by straightforward application of (ii) it is easy to prove Hölder continuity and the fact that a quasiconformal mapping of $R^{n}$ into $R^{n}$ is onto.

For more details of the equivalence of the various definitions and historical remarks I refer to [L2] for $n=2$ and to [V2] for general $n$.

\section{Extension}

One central question in the theory of quasiconformal mappings is the extension of a given mapping, for example to the boundary of the domain or as a mapping of higher dimension $n$. It is convenient here to use the fact that the inclusion $R^{n} \hookrightarrow \bar{R}^{n}$ is a conformal mapping when $\bar{R}^{n}$ has the spherical metric. We can therefore freely pass to $\bar{R}^{n}$ whenever this is appropriate. Topological operations are here taken with respect to $\bar{R}^{n}$. I shall first consider the case of a nice boundary.

3.1. Mappings of a half space. Let $H^{n}$ be the upper half space $\left\{x \in R^{n}\right.$ : $\left.x_{n}>0\right\}$ and let $f: H^{n} \rightarrow H^{n}$ be $K$-quasiconformal. Then it follows easily from (ii) in 2.3 that $f$ extends as a homeomorphism $\bar{H}^{n} \rightarrow \bar{H}^{n}$. For suppose there exist sequences $\left(x_{i}\right)$ and $\left(y_{i}\right)$ in $H^{n}$ that converge to a point $z \in \bar{R}^{n-1}$ and the sequences $\left(f\left(x_{i}\right)\right)$ and $\left(f\left(y_{i}\right)\right)$ converge to points $u$ and $v$ in $\bar{R}^{n-1}$ with $u \neq v$. We may assume $z, u, v \in R^{n-1}$ and $\left|f\left(x_{i}\right)-u\right|,\left|f\left(y_{i}\right)-v\right| \leq|u-v| / 4=r$ for all $i$. Write $U=f^{-1} B^{n}(u, r), V=f^{-1} B^{n}(v, r)$, and let $\Gamma$ be the family of paths in $H^{n}$ that join $U$ and $V$. Since $U$ and $V$ are connected and $z \in \bar{U} \cap \bar{V}$, we have $M(\Gamma)=\infty$. But $M(f \Gamma) \leq \infty$ which contradicts (ii). This implies that $f$ extends as a continuous mapping $\bar{H}^{n} \rightarrow \bar{H}^{n}$. As $f^{-1}$ is also quasiconformal the extension is a homeomorphism. We may now assume that $\infty$ is fixed on the boundary.

Furthermore, $f$ extends as a $K$-quasiconformal mapping $f^{*}: \bar{R}^{n} \rightarrow \bar{R}^{n}$ by reflection. In the proof it is convenient to use the analytic definition and the only point that needs a little checking is the ACL property across $R^{n-1}$.

Let us next pay attention to the restriction $\varphi=f^{*} \mid R^{n-1}$. It satisfies now an inequality

$$
H(x, r) \leq C=C(n, K)
$$

for all $x \in R^{n-1}$ and $r>0$ (see the proof of the first part of (C) in 2.2). Consequently,

$$
H(x) \leq C,
$$


and $\varphi$ is $C$-quasiconformal if $n \geq 3$.

3.2. The case $n=2$. The inequality (a) expresses the fact that $\varphi$ is $C$-quasisymmetric. While (b) is rather useless for $n=2$, the inequality (a) is important and we write it in the form

$$
C^{-1} \leq \frac{\varphi(x+r)-\varphi(x)}{\varphi(x)-\varphi(x-r)} \leq C .
$$

The famous result by Beurling and Ahlfors [B1] says that (c) is a necessary and sufficient condition for a homeomorphism $\varphi: R^{1} \rightarrow R^{1}$ to be a boundary correspondence of a quasiconformal mapping of $H^{2}$ onto itself. The necessity was indicated above. The sufficiency in their paper is given by the following explicit formula for the mapping $f: H^{2} \rightarrow H^{2}$ in terms of $\varphi$ :

$$
f(x)=\left(\frac{1}{2 x_{2}} \int_{0}^{x_{2}}\left(\varphi\left(x_{1}+t\right)+\varphi\left(x_{1}-t\right)\right) d t, \frac{1}{2 x_{2}} \int_{0}^{x_{2}}\left(\varphi\left(x_{1}+t\right)-\varphi\left(x_{1}-t\right)\right) d t\right) .
$$

The extension $f$ is $K(C)$-quasiconformal, it is $C^{1}$, and it is bilipschitz in the hyperbolic metric. Moreover, $K(C) \rightarrow 1$ as $C \rightarrow 1$.

Let $f: H^{2} \rightarrow H^{2}$ again be any quasiconformal mapping that fixes $\infty$. The boundary mapping $\varphi: R^{1} \rightarrow R^{1}$ need not be absolutely continuous. This was shown also in [B1]. However, for $n \geq 3$ the boundary mapping is absolutely continuous with respect to the Lebesgue $(n-1)$-measure because then it is quasiconformal.

If $G$ is a Möbius group acting on $H^{2}$, and $\varphi: \bar{R}^{1} \rightarrow \bar{R}^{1}$ is a $C$-quasisymmetric mapping compatible with $G$, i.e. $\varphi \circ g \circ \varphi^{-1}$ is a Möbius transformation for all $g \in G$, then there exists a quasiconformal extension $f: H^{2} \rightarrow H^{2}$ which is also compatible with $G$. This was first proved by Tukia [T4]. Douady and Earle [D1] gave another proof and their extension $f_{\varphi}$ is conformally natural in the sense that $g \circ f_{\varphi} \circ h=f_{g \circ \varphi \circ h}$ for all Möbius transformations $g, h$ acting on $H^{2}$.

For $n \geq 3$ it is true that a quasiconformal mapping $\varphi: R^{n-1} \rightarrow R^{n-1}$ has a quasiconformal extension $f: H^{n} \rightarrow H^{n}$. This was for $n=3$ proved by Ahlfors [A4], for $n=4$ by Carleson [C1], and finally for general $n$ by Tukia and Väisälä [T7]. In [T7] an important role is played by the paper [S1] by Sullivan.

3.3. Quasicircles. Let us now consider extension problems for more general domains. The case $n=2$ is well understood. Let $D \subset R^{2}$ be a Jordan domain and $f$ a quasiconformal mapping of $D$ onto the unit disk $B^{2}$. By the same argument as before $f$ extends as a homeomorphism $\bar{D} \rightarrow \bar{B}^{2}$. If $f$ has a quasiconformal extension $f^{*}: \bar{R}^{2} \rightarrow \bar{R}^{2}$, then $\partial D$ is called a quasicircle. A surprisingly simple 
characterization of quasicircles was given by Ahlfors [A3]: A Jordan curve $\gamma$ in $R^{2}$ is a quasicircle if and only if there exists a constant $c$ such that

$$
\min _{i=1,2} \operatorname{dia}\left(\gamma_{i}\right) \leq c|x-y|
$$

for all $x, y \in \gamma$ where $\gamma_{1}$ and $\gamma_{2}$ are the components of $\gamma \backslash\{x, y\}$. There exists a number of other characterizations of quasicircles, and quasicircles have many connections to other branches of mathematics, see [G5]. Quasicircles can have any Hausdorff dimension in the interval [1,2[. A typical example of a quasicircle with Hausdorff dimension greater than 1 is the Koch curve, which is constructed as follows. Start with an equilateral triangle, replace the middle third of each side by two line segments so that they together with the middle third form an equilateral triangle whose interior does not meet the interior of the original triangle, and continue similarly.

3.4. Domains quasiconformally equivalent to balls. Let us now turn to the case $n \geq 3$. The situation there is in many respects different and many open problems remain. Let again $D \subset R^{n}$ be a Jordan domain and $f: D \rightarrow B^{n}$ a quasiconformal mapping. We say then that $D$ is quasiconformally equivalent to $B^{n}$. By changing partly the earlier argument one can still show that $f$ extends to a homeomorphism $\bar{D} \rightarrow \bar{B}^{n}$. It was proved quite recently by Kuusalo [K1] that a quasiconformal mapping $D$ onto another Jordan domain need not have a homeomorphic extension to the closure for $n \geq 3$ although such a mapping extends homeomorphically in the case $n=2$.

A result concerning the extension of $f: D \rightarrow B^{n}$ quasiconformally to $\bar{R}^{n}$ is the following [G3], [V4]: $f: D \rightarrow B^{n}$ has an extension to a quasiconformal mapping $f^{*}: \bar{R}^{n} \rightarrow \bar{R}^{n}$ if and only if $\bar{R}^{n} \backslash \bar{D}$ is quasiconformally equivalent to $B^{n}$. This result is not true for $n=2$ since all Jordan domains are for $n=2$ even conformally equivalent to the disk $B^{2}$.

In this connection there are two important open problems for $n \geq 3$. First, to characterize the Jordan domains that are quasiconformally equivalent to $B^{n}$, and second, to characterize quasispheres, that is, images of the unit sphere $S^{n-1}$ under a quasiconformal mapping of $\bar{R}^{n}$ onto itself. The paper [G9] by Gehring and Väisälä is the basic source for the study of the first question. From [G9] are for example the following facts. If we deform a ball by adding to it an outward pointing spire with zero angle, we obtain a domain like an onion (for $n=3$ ). Such a domain is quasiconformally equivalent to $B^{n}$. If we delete such a spire which points inward, we obtain a domain which is not quasiconformally equivalent to $B^{n}$. The role of outward and inward changes if we operate with a wedge of zero angle. Recently Väisälä has given interesting characterizations for cylindrical domains to be quasiconformally equivalent to $B^{3}$ [V5]. No general necessary and sufficient metric condition is known for either problem. 
An interesting topological 2-sphere which is not a quasisphere is obtained by first applying the operations in the construction of the Koch curve to $\bar{R}^{1}$ to obtain a quasicircle $\gamma_{0}$ in $\bar{R}^{2}$. Then we take the product $\left(\gamma_{0} \backslash\{\infty\}\right) \times R^{1}$ which is a surface in $R^{3}$ and add $\infty$ to obtain our topological sphere $Y$ in $\bar{R}^{3}$. Tukia [T3] used this $Y$ and its higher dimensional analogue to show that a quasiconformal group acting on $\bar{R}^{n}$, i.e. a group of $K$-quasiconformal mappings for some $K$, need not for $n \geq 3$ be a conjugation of a Möbius group by a quasiconformal (in fact not even a topological) mapping. For $n=2$ such a group is always a conjugation of a Möbius group by a quasiconformal mapping. This was shown by Sullivan [S2] and Tukia [T2].

\section{Measurable Riemann mapping theorem}

In this section $n=2$ and we consider only sense-preserving quasiconformal mappings, i.e. with Jacobian determinant positive a.e. Let $f: D \rightarrow D^{\prime}$ be $K$ quasiconformal. With the complex notation $z=x+i y$ we have the complex derivatives $f_{x}=\left(f_{x}-i f_{y}\right) / 2, f_{\bar{z}}=\left(f_{x}+i f_{y}\right) / 2$ defined a.e. The $K$-quasiconformality means then that the complex dilatation

$$
\mu_{f}=\frac{f_{\bar{z}}}{f_{z}}
$$

is defined a.e. as a measurable function with $\left\|\mu_{f}\right\|_{\infty} \leq k=(K-1) /(K+1)$. Now the remarkable thing is that the complex dilatation can be given as an arbitrary measurable function with $L^{\infty}$-norm $<1$. This is the measurable Riemann mapping theorem: Let $\mu: \mathbf{C} \rightarrow \mathbf{C}$ be a measurable function with $\|\mu\|_{\infty}<1$. Then there exists a quasiconformal mapping $f: \mathbf{C} \rightarrow \mathbf{C}$ with $\mu_{f}=\mu$ a.e. Moreover, if $f$ is fixed at two points in $\mathbf{C}$, it is unique and it depends holomorphically on $\mu$.

In this form the theorem is proved by Ahlfors and Bers in [A7]. The theorem except for the holomorphic dependence on $\mu$ was proved first by Morrey [M2]. Bojarski [B2] gave a proof where he applied the Calderon-Zygmund theory of singular integrals. This theory was also used in [A7]. In his proof arrangement Bojarski also gets in a natural way the result that the derivative of a quasiconformal mapping belongs locally not only to $L^{2}$ but to $L^{p}$ for some $p>2$. For a long time it was an open question whether such a result is true for quasiconformal mappings in the case $n \geq 3$. This question was answered in the affirmative by Gehring [G4]. Gehring's ideas became quickly an important tool in the theory of partial differential equations.

There are a number of applications of the measurable Riemann mapping theorem. The main application is in the study of Teichmüller spaces. Sullivan has succesfully applied it to the studies of iteration, see e.g. [S3], [S4]. It is also used in the proof of the result mentioned in the preceding section that a quasiconformal 
group for $n=2$ is always a conjugation of a Möbius group by a quasiconformal mapping.

The theorem can also be applied to show that a given $K$-quasiconformal mapping $f$ can be written as

$$
f=g_{1} \circ \cdots \circ g_{m}
$$

where each $g_{j}$ is $K^{1 / m}$-quasiconformal. In dimensions $n \geq 3$ it is an open problem whether a given quasiconformal mapping can be written as a composition of mappings with smaller dilatation. The counterpart for the Beltrami equation $f_{\bar{z}}=\mu f_{z}$ is for $n \geq 3$ an overdetermined system and therefore such a powerful tool as the measurable Riemann mapping theorem is missing.

\section{Further applications and comments}

In addition to those mentioned in earlier sections quasiconformal mappings have a large variety of important applications and connections to other fields of mathematics.

First, quasiconformal mappings can be characterized as the class that preserve the BMO functions [R1], [A8]. For $n=2$ the extension of BMO-functions is tied to quasiconformal theory: If $D$ is a simply connected hyperbolic domain in $R^{2}$, then each BMO function in $D$ has a BMO extension to $R^{2}$ if and only if $\partial D$ is a quasicircle [J1].

Also bilipschitz extensions are closely connected to quasiconformality. Let $n \neq 4$ and $f: E \rightarrow E^{\prime}$ be bilipschitz where $E, E^{\prime} \subset R^{n}$. Then $f$ has a bilipschitz extension to $R^{n}$ if and only if $f$ has a quasiconformal extension to $R^{n}$ [T8]. In the plane a bilipschitz mapping of a Jordan curve $C$ has a bilipschitz extension to $R^{2}$ if and only if $C$ is a quasicircle [G6].

Quasiconformal mappings have played an important role also in some problems in differential geometry. For $n=2$ we have the Teichmüller theory where one studies hyperbolic metrics on surfaces. For $n \geq 3$ a famous application is Mostow's proof of his rigidity theorem [M3] which for compact manifolds can be formulated as follows: If $M$ and $M^{\prime}$ are compact diffeomorphic hyperbolic $n$ manifolds, $n \geq 3$, then $M$ and $M^{\prime}$ are isometric. This result shows that there is no Teichmüller theory for compact manifolds (without boundary) for $n \geq 3$. In the proof two facts are needed from quasiconformal theory. First, that a quasiconformal self mapping of $H^{n}$ extends as a quasiconformal mapping to the boundary (cf. 2.1), and second, that a quasiconformal mapping is differentiable a.e. The theorem has later been generalized in various directions for example by Agard, Mostow, Sullivan, and Tukia.

In the proof of the conjecture of Lichnerowicz [L3], which states that the group of conformal self mappings of a compact Riemannian $n$-manifold $M, n \geq 2$, is compact provided $M$ is not conformally equivalent to the standard sphere, quasiconformal theory was also used. 
A result of Sullivan [S1] says that for $n \neq 4$ every topological manifold has a quasiconformal structure, i.e. overlap transformations can be chosen quasiconformal, and this structure is unique up to homeomorphisms arbitrary close to identity. Sullivan shows the same for Lipschitz structures. See also [T6]. Recently Donaldson and Sullivan (see [S4]) have studied the case $n=4$ and found that the situation for quasiconformal structures is much like the smooth case where Donaldson and Freedman recently established their sensational results: Many topological 4-manifolds do not have quasifonformal structures and many pairs of homeomorphic 4-manifolds exist with quasiconformal structures essentially different.

In 3.4 I mentioned Tukia's result [T3] that for $n \geq 3$ there are quasiconformal groups acting on $\bar{R}^{n}$ that are not quasiconformally conjugate to a Möbius group (although for $n=2$ this is the case). Consequently this has increased the interest in the study of quasiconformal groups, see [G8], [T5], [F1]. In particular, it is important in the case $n \geq 3$ to give conditions for a quasiconformal conjugacy to a Möbius group. Results in this direction are e.g. in the papers by Gromov [G10, p. 209], Tukia [T5, p. 340], and Sullivan [S4, Part IV].

\section{Noninjective mappings}

If we let $f: D \rightarrow R^{n}$ be a continuous mapping that satisfies for some $K^{\prime} \in$ $[1, \infty[$

(a) $f$ is $A C L^{n}$,

(b) $\left|f^{\prime}(x)\right|^{n} \leq K^{\prime} J_{f}(x)$ a.e.,

we call $f$ quasiregular. We see that this definition is obtained from the analytic definition of quasiconformal mappings (see 2.1 and 2.3) by replacing the word homeomorphism by continuous and by requiring $J_{f}(x) \geq 0$ a.e. Like quasiconformal mappings quasiregular mappings are well defined also on oriented Riemannian $n$-manifolds. Quasiregular mappings form a particularly interesting class, and it has turned out that these mappings give the right generalization of the geometric part of the theory of complex analytic functions in the plane to real $n$-dimensional space.

Quasiregular mappings were first introduced and studied by Reshetnyak in a series of papers in the late 1960's and his contributions are presented in the book [R3]. Some years later Martio, Rickman, and Väisälä started a study with a somewhat more geometric point of view on the subject, see [M1], [V3]. See also Vuorinen [V6].

The situation for $n=2$ is in the Euclidean case the following. The 1quasiregular mappings are exactly the analytic functions. If $f$ is quasiregular, then it is of the form $f=g \circ h$ where $h$ is quasiconformal and $g$ analytic [L2, Chapter VI]. Because of this decomposition, the quasiregular mappings for $n=2$ have relatively little independent interest.

For $n \geq 3$ the situation is different. First, the 1-quasiregular mappings are also now restrictions of Möbius transformations. One of Reshetnyak's main results 
is that a nonconstant quasiregular mapping $f$ is discrete (i.e. $f^{-1}(y)$ consists of isolated points) and open, and $f$ shares thus these topological properties with the planar analytic functions. The simplest examples are finite to one branched covers.

In 1967 Zorich [Z1] proved that a locally homeomorphic quasiregular mapping of $R^{n}$ into itself is always a homeomorphism if $n \geq 3$. This phenomenon was anticipated already by Lavrent'ev in 1938 [L1]. It shows a certain rigidity for $n \geq 3$ and therefore interesting quasiregular mappings for $n \geq 3$ have in general branching. In his paper Zorich also posed the question of the validity of a Picard's theorem on omitted values and this problem was for a long time one of the main open questions in the theory of quasiregular mappings. In [R4] a Picard type theorem was proved in the following form: There exist an integer $q=q(n, K)$ such that every $K$-quasiregular mapping $f: R^{n} \rightarrow R^{n} \backslash\left\{a_{1}, \ldots, a_{q}\right\}$ is constant. This is known to be qualitatively sharp for $n=3$ [R6]: For every positive integer there exists a quasiregular mapping $f: R^{3} \rightarrow R^{3}$ omitting $p$ points in $R^{3}$. The case $p=1$ is easy and the construction was given in [Z1]. The sharpness for $n \geq 4$ is an open question.

Value distribution for quasiregular mappings in the spirit of Ahlfors' paper [A1] on covering surfaces is also understood to a certain extent. A recent result [R7] says that a sharp analogue for a pointwise defect relation is true for quasiregular mappings. To state this let $f: R^{n} \rightarrow \bar{R}^{n}$ be a nonconstant $K$-quasiregular mapping, let $n(r, y)$ be the cardinality of the set $f^{-1}(y) \cap \bar{B}^{n}(r)$ with multiplicity, and let $A(r)$ be the average of $n(r, y)$ when $y$ runs over the points in $\bar{R}^{n}$. Then there exists a set $E \subset\left[1, \infty\left[\right.\right.$ of finite logarithmic measure, i.e. $\int_{E} d r / r<\infty$, such that

$$
\limsup _{\substack{r \rightarrow \infty \\ r \notin E}} \sum_{j=1}^{q}\left(1-\frac{n\left(r, a_{j}\right)}{A(r)}\right)_{+} \leq C(n, K)
$$

whenever $a_{1}, \ldots, a_{q}$ are distinct points in $\bar{R}^{n}$. In Ahlfors' theory the bound is 2 for $n=2$. A weaker theorem was proved in [R5]. The result (1) is qualitatively sharp for $n=3$ in the sense that arbitrarily given defect numbers $\delta_{j}$ can be asymptotically realized with proper constraints [R7]. More precisely, given points $a_{1}, a_{2}, \ldots \in \bar{R}^{3}$ and numbers $\delta_{1}, \delta_{2}, \ldots$ such that $0 \leq \delta_{j} \leq 1$ and $\sum_{j} \delta_{j} \leq P$, then there exists a $K$-quasiregular mapping $f: R^{3} \rightarrow \bar{R}^{3}$ such that

$$
\begin{aligned}
& \lim _{r \rightarrow \infty}\left(1-\frac{n\left(r, a_{j}\right)}{A(r)}\right)=\delta_{j}, \quad j=1,2, \ldots, \\
& \lim _{r \rightarrow \infty}\left(1-\frac{n(r, y)}{A(r)}\right)=0 \quad \text { if } \quad y \notin\left\{a_{1}, a_{2}, \ldots\right\},
\end{aligned}
$$

where $K$ depends only on $P$. Cf. Drasin's corresponding result in [D2] for meromorphic functions. The sharpness for $n \geq 4$ is open. 
Reshetnyak's work on the subject is centered around nonlinear potential theory and he gives counterparts for harmonic functions to quasiregular theory. His studies have been extended recently by Martio and his students. However, like for quasiconformal mappings, the most effective tool for the study of quasiregular mappings has turned out to be the method of moduli of path families. Of the quasiinvariance 2.3(ii) only the right hand inequality, known as Poletski1̌s inequality, is true for quasiregular mappings. The heart of the matter is to combine estimates for the modulus properly with covering properties of the mapping.

One interesting area of research is to describe Riemannian $n$-manifolds $M$ and $N$ for which there exists a nonconstant quasiregular mapping of $M$ into $N$. The Picard type theorem mentioned above belongs to this area. If $N=S^{n}$, the mapping always exists. To limit the general question let us discuss somewhat the case $M=R^{n}, N$ compact.

Gromov [G11, Chapter VI] and Pansu [P1] have used the connection of isoperimetric inequalities and conformal capacity for this mapping problem. The idea goes back to Ahlfors [A1, p. 188]. For example, let $G$ be the Heisenberg group, i.e. the Lie group of upper triangular $3 \times 3$ matrices with ones on the diagonal and real entries, let $H$ be the subgroup of integer entries, and let us fix a left invariant Riemannian metric on $G$. Then $G$ satisfies an isoperimetric inequality which is stronger than the one for $R^{3}$. It follows that there exists no nonconstant quasiregular mapping of $R^{3}$ into $N=G / H$.

Another type of example is the connected sum $T^{4} \# S^{2} \times S^{2}$ where $T^{4}$ is the 4-torus. After some preparations where both the nontriviality of $\pi_{1}\left(T^{4}\right)$ and $H_{2}\left(S^{2} \times S^{2}\right)$ are used, one can apply the ideas from the proof of the Picard type theorem to show that there is no nonconstant quasiregular mapping $R^{4} \rightarrow$ $T^{4} \# S^{2} \times S^{2}$. On the other hand, such a mapping exists of $R^{4}$ into $S^{2} \times S^{2}$, namely, there exists a two to one branched cover $T^{2} \rightarrow S^{2}$ and then we get the mapping as $R^{4} \rightarrow T^{4}=T^{2} \times T^{2} \rightarrow S^{2} \times S^{2}$. An open problem is whether there exists such a mapping of $R^{4}$ into $S^{2} \times S^{2} \# S^{2} \times S^{2}$.

\section{References}

[A1] Ahlfors, L.V.: Zur theorie der Überlagerungsflächen. - Acta Math. 65, 1935, 157-194.

[A2] Ahlfors, L.V.: On quasiconformal mappings. - J. Analyse Math. 3, 1953/54, 1-58.

[A3] Ahlfors, L.V.: Quasiconformal reflections. - Acta Math. 109, 1963, 291-301.

[A4] Ahlfors, L.V.: Extension of quasiconformal mappings from two to three dimensions. Proc. Nat. Acad. Sci. U. S. A. 51, 1964, 768-771.

[A5] AhLFors, L.V.: Lectures on quasiconformal mappings. - Van Nostrand Company, TorontoNew York-London, 1966.

[A6] Ahlfors, L.V.: Quasiconformal mappings, Teichmüller spaces, and Kleinian groups. Proceedings of the International Congress of Mathematicians, Helsinki, 1978, 71-84.

[A7] Ahlfors, L.V., and L. Bers.: Riemann's mapping theorem for variable metrics. - Ann. of Math. 72, 1960, 385-404. 
[A8] Astala, K.: A remark on quasi-conformal mappings and BMO-functions. - Michigan Math. J. 30, 1983, 209-212.

[B1] Beurling, A., and L.V. AhlFors: The boundary correspondence under quasiconformal mappings. - Acta Math. 96, 1956, 125-142.

[B2] BojARski, B.: Generalized solutions of a system of first order differential equations of elliptic type with discontinuous coefficients. - Mat. Sb. 43, 1957, 451-503 (Russian).

[C1] CARLESON, L.: The extension problem for quasiconformal mappings. - Contributions to analysis, Academic Press, New York-London, 1974, 39-47.

[D1] DouAdY, A., and C.J. EARLE: Conformally natural extension of homeomorphisms of the circle. - Acta Math. 157, 1986, 23-48.

[D2] Drasin, D.: The inverse problem of the Nevanlinna theory. - Acta Math. 138, 1977, 83151.

[F1] Freedman, M.H., and R. Skora: Strange actions of groups on spheres, - J. Differential Geom. 25, 1987, 75-98.

[G1] Gehring, F.W.: Symmetrization of rings in space. - Trans. Amer. Math. Soc. 101, 1961, 499-519.

[G2] Gehring, F.W.: Rings and quasiconformal mappings in space. - Trans. Amer. Math. Soc. 103, 1962, 353-393.

[G3] Gehring, F.W.: Extension of quasiconformal mappings in three space. - J. Analyse Math. $14,1965,171-182$.

[G4] Gehring, F.W.: The $L^{p}$-integrabillity of the partial derivatives of a quasiconformal mapping. - Acta Math. 130, 1973, 265-277.

[G5] Gehring, F.W.: Characteristic properties of quasidisks. - Les Presses de l'Université de Montréal, 1982.

[G6] Gehring, F.W.: Extension of quasiisometric embeddings of Jordan curves. - Complex Variables 5, 1986, 245-263.

[G7] Gehring, F.W.: Topics in quasiconformal mappings. - Proceedings of the International Congress of Mathematicians, Berkeley 1986, American Mathematical Society, Providence, R. I.,1987, 62-80.

[G8] Gehring, F.W., and G.J. Martin: Discrete quasiconformal groups I. - J. London Math. Soc. (3) 55, 1987, 331-358.

[G9] Gehring, F.W., and J. VÄISÄLÄ: The coefficients of quasiconformality of domains in space. - Acta Math. 114, 1965, 1-70.

[G10] Gromov, M.: Hyperbolic manifolds, groups and actions. - Riemann surfaces and related topics: Proceedings of the 1978 Stony Brook Conference. Annals of Math. Studies 97, Princeton University Press, Princeton, 1981, 183-213.

[G11] Gromov, M.: Structures métriques pour les variétés riemanniennes. - Notes de cours rédigées par L. Lafontaine et P. Pansu, CEDIC-Fernand-Nathan, Paris, 1981.

[G12] Grötzsch, H.: Über möglichst konforme Abbildungen von schlichten Bereichen. - Ber. Verh. Sächs. Akad. Wiss. Leipzig 84, 1932, 114-120.

[J1] Jones, P.W.: Extension theorems for BMO. - Indiana Univ. Math. J. 29, 1980, 41-46.

[K1] KuUsalo, T.: Quasiconformal mappings without boundary extensions. - Ann. Acad. Sci. Fenn. Ser. A I Math. 10, 1985, 331-338.

[L1] Lavrent'ev, M.A.: Sur un critère différentiel des transformations homéomorphes des domaines à trois dimensions. - Dokl. Akad. Nauk. 20, 1938, 241-242.

[L2] Lehto, O., and K.I. Virtanen: Quasiconformal mappings in the plane. - SpringerVerlag, Berlin-Heidelberg-New York, 1973. 
[L3] LeLONG-FerRand, J.: Transformations conformes et quasi-conformes des variétés riemanniennes compactes (Démonstration de la conjecture de A. Lichnerowicz). - Acad. Roy. Belg. Cl. Sci. Mém. Coll. 39, 1971, 1-44.

[L4] Loewner, C.: On the conformal capacity in space. - J. Math. Mech. 8, 1959, 411-414.

[M1] Martio, O., S. Rickman, and J. VÄISÄLÄ: Topological and metric properties of quasiregular mappings. - Ann. Acad. Sci. Fenn. Ser. A I Math. 488, 1971, 1-31.

[M2] Morrey, C.B.: On the solutions of quasi-linear elliptic partial differential equations. Trans. Amer. Math. Soc. 43, 1938, 126-166.

[M3] Mostow, G.D.: Quasi-conformal mappings in $n$-space and the rigidity of hyperbolic space forms. - Publ. Math. Institut des Hautes Études Sci. 34, 1968, 53-104.

[P1] PANSU, P.: An isometric inequality on the Heisenberg group. - Proceedings on Differential Geometry on Homogeneous Spaces, Torino, 1983, 159-174.

[R1] ReimanN, H.M.: Functions of bounded mean oscillation and quasiconformal mappings. Comment. Math. Helv. 49, 1974, 260-276.

[R2] ReshetNyAK, YU.G.: Liouville's theorem on conformal mappings under minimal regularity assumptions. - Sibirsk. Math. Zh. 8, 1967, 835-840 (Russian).

[R3] ReshetNyak, YU.G.: Spatial mappings with bounded distortion. - Izdatel'stvo "Nauka", Sibirsk. Otdelenie, Novosibirsk, 1982 (Russian).

[R4] Rickman, S.: On the number of omitted values of entire quasiregular mappings. - J. Analyse Math. 37, 1980, 100-117.

[R5] Rickman, S.: A defect relation for quasimeromorphic mappings. - Ann. of Math. 114, 1981, 165-191.

[R6] Rickman, S.: The analogue of Picard's theorem for quasiregular mappings in dimension three. - Acta Math. 154, 1985, 195-242.

[R7] RICKMan, S.: Defect relation and its realization for quasiregular mappings. - In preparation.

[S1] Sullivan, D.: Hyperbolic geometry and homeomorphisms. - Geometric topology, Academic Press, New York-London, 1979, 543-555.

[S2] Sullivan, D.: On the ergodic theory at infinity of an arbitrary discrete group of hyperbolic motions. - Riemann surfaces and related topics: Proceedings of the 1978 Stony Brook Conference, Annals of Math. Studies 97, Princeton University Press, Princeton, 1981, 465-496.

[S3] Sullivan, D.: Quasiconformal homeomorphisms and dynamics I. Solution of the FatouJulia problem on wandering domains. - Ann. of Math. 122, 1985, 401-418.

[S4] Sullivan, D.: Quasiconformal homeomorphisms in dynamics, topology, and geometry. Proceedings of the International Congress of Mathematicians, Berkeley 1986, American Mathematical Society, Providence, R. I., 1987, 1216-1228.

[T1] TeIChmüLleR, O.: Extremale quasiconforme Abbildungen und quadratische Differentiale. - Abh. Preuss. Akad. Wiss. 22, 1940, 1-197.

[T2] Tukia, P.: On two-dimensional quasiconformal groups. - Ann. Acad. Sci. Fenn. Ser. A I Math. 5, 1980, 73-78.

[T3] Tukia, P.: A quasiconformal group not isomorphic to a Möbius group. - Ann. Acad. Sci. Fenn. Ser. A I Math. 6, 1981, 149-160.

[T4] Tukia, P.: Quasiconformal extension of quasisymmetric mappings compatible with a Möbius group. - Acta Math. 154, 1985, 153-193.

[T5] TukiA, P.: On quasiconformal groups. - J. Analyse Math. 46, 1986, 318-346. 
[T6] TUKIA, P., and J. VÄISÄLÄ: Lipschitz and quasiconformal approximation and extension. - Ann. Acad. Sci. Fenn. Ser. A I Math. 6, 1981, 303-342.

[T7] TukIA, P., and J. V̈̈̈Ï̈̈L̈̈: Quasiconformal extension from dimension $n$ to $n+1$. Ann. of Math. 115, 1982, 331-348.

[T8] TUKIA, P., and J. VÄISÄL̈̈: Bilipschitz extensions of maps having quasiconformal extensions. - Math. Ann. 269, 1984, 561-572.

[V1] VÄISÄLÄ, J.: On quasiconformal mappings in space. - Ann. Acad. Sci. Fenn. Ser. A I Math. 298, 1961, 1-36.

[V2] VÄISÄL̈̈, J.: Lectures on $n$-dimensional quasiconformal mappings. - Lecture Notes in Mathematics 229. Springer-Verlag, Berlin-Heidelberg-New York, 1971.

[V3] VÄISÄL $\ddot{A}$, J.: A survey of quasiregular maps in $R^{n}$. - Proceedings of the International Congress of Mathematicians, Helsinki, 1978, 685-691.

[V4] VÄISÄLÄ, J.: Quasimöbius maps. - J. Analyse Math. 44, 1984/85, 218-234.

[V5] VÄISÄLÄ, J.: Quasiconformal maps of cylindrical domains. - Acta Math. (to appear).

[V6] VuoRinen, M.: Conformal geometry and quasiregular mappings. - Lecture Notes in Mathematics 1319. Springer-Verlag, Berlin-Heidelberg-New York, 1988.

[Z1] ZoRICH, V.A.: The theorem of M.A. Lavrent'ev on quasiconformal mappings in space. Mat. Sb. 74, 1967, 417-433 (Russian).

University of Helsinki

Department of Mathematics

Hallituskatu 15

SF-00100 Helsinki

Finland

Received 10 May 1988 